\title{
Pleuramesotheliom - Pathologie und Pathogenese
}

\author{
K.-M. Müller
}

\author{
Pleuromesothelioma - Psychology and Pathogenesis
}

\section{Zusammenfassung}

Pathologisch-anatomische Untersuchungen sind für Diagnose und Therapie pleuraler Tumoren von entscheidender Bedeutung. Im Vordergrund stehen Abgrenzungen reaktiv-hyperplastischer Mesothelproliferate zu bereits manifesten bösartigen primären oder sekundären pleuralen Tumoren. Bei bisher nicht vorhandenen spezifischen morphologischen und molekularbiologischen Befunden für Pleuramesotheliome ergeben aber gezielt eingesetzte immunhistochemische und zytometrische Zusatzuntersuchungen wertvolle Hinweise für die differenzialdiagnostische Unterscheidung primärer und sekundärer pleuraler Neoplasien. Für operative Maßnahmen unter kurativer Zielsetzung ist die Tumordiagnose in frühen Entwicklungsphasen von Mesothelioma in situ und Frühmesotheliomen durch eine frühzeitige invasive Diagnostik dringend notwendig. Der Pathologe ist bei der aufgezeigten Problematik der Tumorsicherung aber auf ausreichendes Untersuchungsgut angewiesen. 90\% der Pleuramesotheliome sind als meist beruflich bedingte Asbest-assoziierte Tumoren zu werten und als Verdachtsfälle im Sinne beruflich verursachter Krebserkrankungen bei einer Berufsgenossenschaft anzeigepflichtig. Bei nicht selten problematischen Befunden der pathologisch-anatomischen Tumorsicherung steht das Deutsche Mesotheliomregister in Bochum - gefördert vom Hauptverband der gewerblichen Berufsgenossenschaften - als Konsiliareinrichtung zur Verfügung.

\section{Abstract}

Investigations into the anatomical pathology are of crucial importance for the diagnosis and therapy of pleural tumours. The main objective is to differentiate reactive hyperplastic proliferations of the mesothelium from already manifest primary or secondary malignant pleural tumours. Given the absence of distinct morphological features displayed by pleural mesotheliomas, specific additional immunohistochemical and cytometric tests can provide valuable clues for the differential diagnosis of primary and secondary pleural neoplasms. Confirmation of the diagnosis during the initial stages of tumour development i.e. mesothelioma in-situ and early mesothelioma necessitates invasive diagnostic procedure as early as possible. This holds particularly true if surgery is aimed at cure. However, in order to firmly establish the diagnosis considering the afore mentioned problems, the tissue specimens to be examined by a pathologist must be of adequate size. $90 \%$ of all pleural mesotheliomas are asbestos associated due to occupational exposure. Therefore, all cases of a suspected pleural mesothelioma must be reported to the appropriate professional association as a potential occupational disease. The Bochum based German Mesothelioma Registry, which is supported by the head office of the German Professional Associations, conducts research and acts as an advisory institution in difficult cases, where problems concerning the classification of tumours by their anatomical pathology arise.

Serienherausgeber: $R$. Loddenkemper, W. Frank

Institutsangaben

Institut für Pathologie, Kliniken Bergmannsheil, Bochum (Direktor: Prof. Dr. med. K.-M. Müller)

Anmerkung

Mit Unterstützung des Hauptverbandes der gewerblichen Berufsgenossenschaften e.V., St. Augustin

Korrespondenzadresse

Prof. Dr. K.-M. Müller · Institut für Pathologie · Kliniken Bergmannsheil · Bürkle-de-la-Camp-Platz 1

44789 Bochum ·E-mail: patho-bhl@ruhr-uni-bochum.de

Bibliografie

Pneumologie 2004; 58: 670-679 • (c) Georg Thieme Verlag KG Stuttgart · New York

DOI $10.1055 / S-2004-818505$

ISSN 0934-8387 


\section{Vorbemerkungen}

Die Differenzialdiagnose primärer und sekundärer Pleuratumoren kann ausgesprochen problematisch sein. Beim histogenetisch begründbaren sehr vielfältigen histologischen und zytologischen Bild können grundsätzlich bei primären Mesotheliomen und sekundären Pleurakarzinomen phänotypisch sehr ähnliche Befunde resultieren. Die Zusammenhänge zwischen einer erhöhten meist beruflichen, auch viele Jahre zurückliegenden Asbeststaubexpostition und der Entwicklung pleuraler und abdomineller Mesotheliome ist besonders durch die Arbeiten von Wagner u. Mitarb. und die Erfahrungen der letzten 50 Jahre auch epidemiologisch eindeutig belegt (Wagner u. Mitarb. 1960 [14]). Diese Untersuchungsergebnisse begründen die Einordnung der primären Mesotheliome bei beruflich bedingten Krebserkrankungen (Literatur siehe Jones JSP u. Mitarb. 1987 [4]; Müller K-M 1997 [10]; Krismann M und Müller K-M 2000 [8]).

\section{Pleuramesotheliom}

Die Geschichte der malignen Mesotheliome in der Medizin ist noch jung. Erst in den 20er Jahren des 20. Jahrhunderts konnte die Existenz primärer Tumoren des Lungen-Rippenfells bewiesen werden. Eine zuverlässige Diagnostik insbesondere bei der Abgrenzung von sekundären Tumoren der serösen Häute ist erst seit den 80er Jahren mit ständigen Fortschritten in der Immunhistochemie möglich geworden (Literatur siehe Krismann M u. Mitarb. 2000 [8]).

Seit etwa 1985 kam es im Rahmen der Zunahme asbestassoziierter Mesotheliome zu einem erheblichen Anstieg der Inzidenz. Derzeit werden im deutschen Mesotheliomregister in Bochum jährlich mehr als 600 neue Erkrankungsfälle gesichert (Abb.1).

Hinzu kommt eine nicht genau bekannte „Dunkelziffer“ nicht zentral erfasster Mesotheliome. Eine weitere Zunahme der Mesotheliome bis etwa 2020 wird prognostiziert. Die Inzidenz der nicht asbestassoziierten malignen Mesotheliome beträgt etwa 1 - 2 pro 1 Mio. Einwohner.

Die pleurale Manifestation ist die bei weitem häufigste (Verhältnis Pleura : Peritoneum etwa 10:1). Das Perikard ist primär nur in etwa $2 \%$ aller Mesotheliome und die Tunica vaginalis testis nur in Einzelfällen betroffen. Aufgrund der überwiegenden beruflichen Asbestexposition sind Männer insgesamt wesentlich häufiger betroffen. Das Hauptmanifestationsalter der Mesothe-

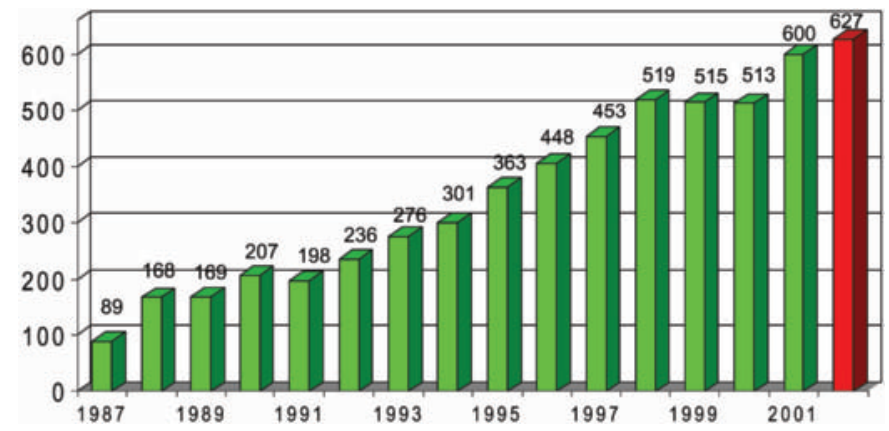

Abb. 1 Anstieg der gesicherten Mesotheliome im Deutschen Mesotheliom-Register in Bochum von 1987-2002. liome liegt im 60. Lebensjahrzehnt. Primäre Tumoren stellen lediglich etwa $1-3 \%$ aller malignen pleural manifestierten Tumoren dar. Bei weitem häufiger sind metastatische, oft pseudomesotheliomatös wachsende Pleurakarzinosen, überwiegend von Adenokarzinomen der Lunge, der Mamma, des Gastrointestinaltrakts, der Ovarien, des Pankreas und der Nieren.

\section{Ätiologie \\ Asbest}

Nach wie vor kommt der überragende Stellenwert bei der kausalen Pathogenese maligner Mesotheliome dem Asbest zu (Abb. 2).

Der Zusammenhang zwischen der Entwicklung maligner Mesotheliome und einer früheren inhalativen Asbestexposition wurde 1960 erstmals dokumentiert. Obwohl der Arbeitsstoff schon seit der Antike bekannt war, kam es technologiebedingt erst nach dem zweiten Weltkrieg zu einem weltweiten Asbestboom. Der maximale Asbestverbrauch in Deutschland fand in den Jahren zwischen 1965 und 1980 statt. Die Latenzzeiten bei der Mesotheliomentwicklung zwischen Expositionsbeginn und Manifestation betragen im Mittel mehr als 30 Jahre. Alle kommerziell verwendeten Asbestarten können Mesotheliome induzieren. Eine Expositions-Schwellendosis für die Entwicklung maligner Mesotheliome ist nicht bekannt. Im Gegensatz zum asbestassoziierten Lungenkrebs können aber auch kurze und gelegentlich vergleichsweise nur geringe Asbestkontakte ausreichen, um nach entsprechender Latenzzeit Mesotheliome zu induzieren. Derzeit sind etwa 90\% der im Deutschen Mesotheliomregister erfassten malignen Mesotheliome asbestassoziiert.

\section{Erionit}

Eine inhalative urbane Exposition gegenüber Erionit, einem in bestimmten Regionen der Türkei vorkommenden nicht-asbestartigen vulkanischen Zeolith, ist ebenfalls mit einem erhöhten Risiko zur Entwicklung maligner Mesotheliome behaftet. Auch im Deutschen Mesotheliomregister wurden mehrere Patienten aus verschiedenen Orten der Türkei registriert, die als Gastarbeiter lange in Deutschland gearbeitet haben und an Mesotheliomen erkrankten. Hierbei muss dann unter Umständen zwischen einer möglichen urbanen und einer beruflichen Exposition differenziert werden (Abb. 3 ).

\section{Künstliche Mineralfasern}

Bei künstlichen Mineralfasern wie Steinwolle oder Schlackenwolle, die als Asbestersatzstoffe eingesetzt werden, handelt es sich um amorphe Silicate mit erheblich größerer Fasergeometrie

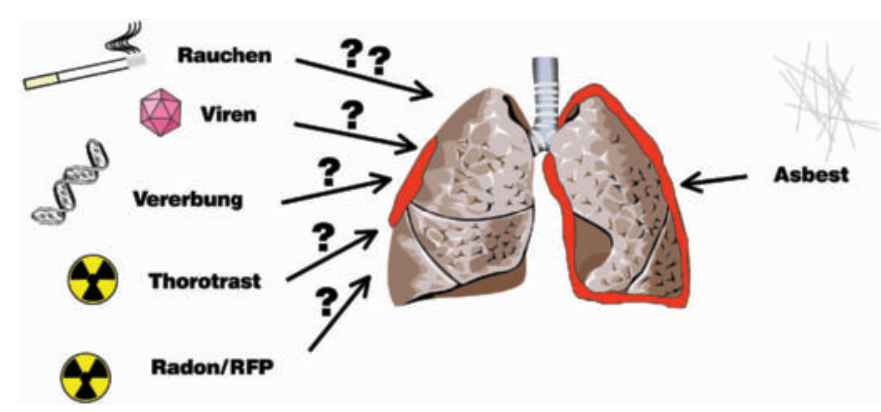

Abb. 2 Übersicht gesicherter und möglicher Kausalfaktoren für die Entwicklung von Pleuramesotheliomen. 


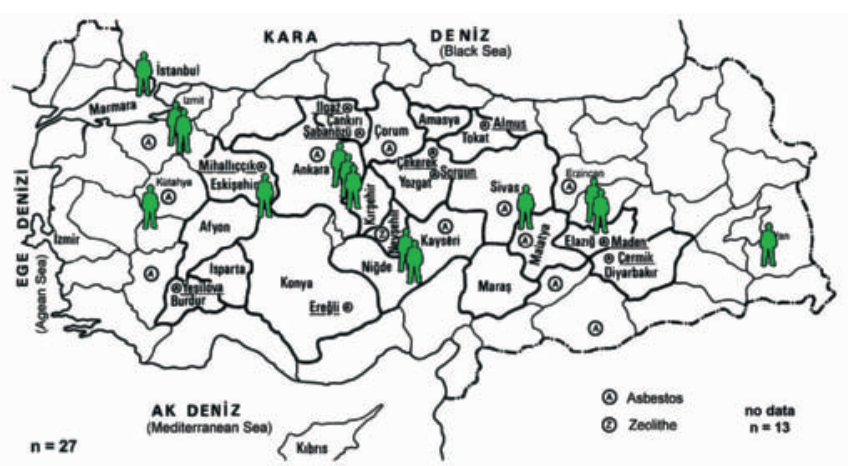

Abb. 3 Übersicht der früheren Wohnorte von Türken in der Türkei, die in Deutschland an einem Mesotheliom erkrankt sind (Daten des Deutschen Mesotheliomregisters).

und einem differenten Bruchverhalten. Eine abschließende Bewertung der möglichen Induktion maligner Mesotheliome durch künstliche Mineralfasern ist derzeit noch nicht möglich. Tierexperimentelle Daten sind widersprüchlich. Es konnten bislang im Tierversuch lediglich nach intraperitonealer Installation Malignome erzeugt werden, nicht jedoch nach inhalativer oder intratrachealer Exposition. Im Deutschen Mesotheliomregister ist derzeit noch kein gesicherter Fall eines Mesothelioms nach ausschließlicher Exposition gegenüber künstlichen Mineralfasern bekannt. Immer wieder wurden auch nicht faserförmige Substanzen in Zusammenhang mit der Induktion maligner Mesotheliome diskutiert. Für das Zigarettenrauchen konnte kein direkter Zusammenhang mit der Entstehung maligner Mesotheliome belegt werden.

Eine familiäre Häufung von Mesotheliomen ist zuweilen zu beobachten. Hierbei handelt es sich oft um Fälle von vermehrt asbestexponierten Familienmitgliedern, deren Angehörige asbestkontaminierte Arbeitskleidung gereinigt haben. Eine epidemiologische Studie konnte bei $71 \%$ der untersuchten Mesotheliompatienten eine vorbekannte Krebserkrankung in der Familie belegen, im Vergleich zu $44 \%$ bei untersuchten Patienten mit malignen Lungentumoren.

\section{Die Diskussion um das Simian Virus 40 (SV 40)}

In humanen Mesotheliomen wurden erstmals 1994 SV 40-ähnliche DNA-Sequenzen nachgewiesen (Light RW 2001 [9]). Positive Ergebnisse bei dem Nachweis von Virussegmenten in malignen Mesotheliomen erzielten in der Folge mehrere Arbeitsgruppen. Die Diskussion über eine mögliche Beteiligung des SV-40-Virus an der Entstehung humaner Mesotheliome erlangte durch die Entdeckung, dass zwischen 1955 und 1963 mit SV 40 kontaminierte Poliomyelitis-Vaccinen in Europa und den USA in Gebrauch waren, schlagartig die Bedeutung einer internationalen Forschungsaufgabe mit hoher sozialmedizinischer Implikation.

Das SV-40-Virus gehört zu den sehr gut untersuchten Viren. In dem 5265 Basenpaaren umfassenden Genom des Papovavirus SV 40 werden ein so genanntes „frühes“ und ein „spätes“ Segment unterschieden. Das frühe Segment kodiert für 2 Proteine, die als klein-t- (tAg) und groß-T-Antigen (TAg) bezeichnet werden. Diese Proteine sind für die Transformation in vitro und die Karzinogenität in vivo verantwortlich. Grundlage der kanzeroge- nen Potenz des SV 40 ist die Fähigkeit des TAg, an die bekannten wachstumsinhibierenden Proteine p53 und die Proteine der Retinoblastom- (Rb)-Familie zu binden und diese funktionell zu inaktivieren. In aktuellen Untersuchungen unter Einschluss von deutschen und US-amerikanischen Proben anhand eines größeren Kollektivs mit 118 Mesotheliomen, 13 reaktiven Veränderungen der Pleura und 20 Vergleichsfällen von malignen Lungentumoren konnten spezifische DNA-Sequenzen für das SV-40-Virus in $57 \%$ der epitheloiden pleuralen und peritonealen Mesotheliome, jedoch in keinem der biphasischen und sarkomatoiden Mesotheliome und in keinem der malignen Lungentumoren nachgewiesen werden. Auch in nicht invasiven Mesotheliomen waren Virussequenzen nachweisbar, nicht jedoch in dem angrenzenden tumorfreien Gewebe, welches in einer Probe zur Untersuchung gelangte (Carbone u. Mitarb. 1994 [2]; Shivapurkar u. Mitarb. 2000 [12]).

\section{Pathogenese}

Die genauen Mechanismen der molekularen Pathogenese maligner Mesotheliome sind trotz vieler vorwiegend experimenteller Daten nur unvollständig bekannt. Wie bei anderen Organtumoren sind offenbar eine Vielzahl von genetischen Alterationen an der Tumorinitiierung und -progression beteiligt. Beim Studium bereits manifester Tumoren sind die genomischen Veränderungen in der Regel so weit fortgeschritten, dass eine sichere Zuordnung zu primären, für die zelluläre Tumorrealisation bedeutsamen und sekundären Läsionen im Rahmen des autonomen Zellwachstums nicht möglich ist (Abb.4).

\section{Genetische Befunde}

Die erste Arbeit über zytogenetische Befunde bei 2 malignen Mesotheliomen stammt aus dem Jahr 1978. Erst 10 Jahre später wurden an Kollektiven von 15 - 30 Fällen weitere zytogenetische Untersuchungen an Mesotheliomen durchgeführt. Zumeist waren komplexe Alterationen vorhanden. Abschnitte der Chromosomen 1, 3 und 7 waren mit interstitiellen oder terminalen Deletionen oder Translokationen involviert. Bereits hier wurde eine Beteiligung der Onkogene myc (Myelocytomatosis Virus-Familie), ras (Rat sarcoma virus), raf (RAS activated fragment) und

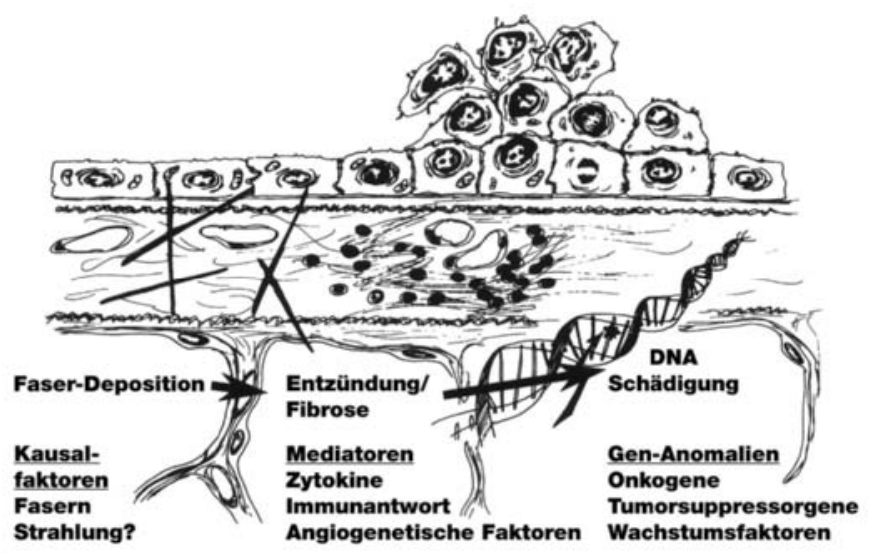

Abb. 4 Schema zur kausalen und formalen Pathogenese maligner Mesotheliome als Reaktion auf jahrelang pleural inkorporierte Asbestfasern. Aktivierung von Onkogenen und Inaktivierung von Tumorsuppressorgenen als mitwirkende Kausalfaktoren bei der Entwicklung pleuraler maligner Tumoren. 
met (N-methyl-N'-nitroso-guanidin treated human osteosarcoma cell line) für die Pathogenese maligner Mesotheliome diskutiert (Literatur siehe Krismann M u. Mitarb. 2002 [7]). Weitere Arbeiten, die Verluste auf Chromosom 1 nachweisen konnten, sind zahlreich. Auf Chromosom 9 konnten Verluste von verschiedenen Arbeitsgruppen teils mit klassischer Zytogenetik, teils mit neueren Methoden auch unter Einschluss der komparativen genomischen Hybridisierung (CGH) belegt werden. Chromosom 14 zeigte bei verschiedenen Untersuchern mit unterschiedlichen Methoden wiederkehrend Verluste. Auch Verluste von Chromosom 22 sind mehrfach beschrieben worden. Das auf Chromosom 17p lokalisierte Tumorsuppressorgen $\mathrm{p} 53$ ist zwar im Tierexperiment häufig von einem Funktionsverlust betroffen, für humanes Tumorgewebe existieren aber kontroverse Mitteilungen.

Eigene CGH-Ergebnisse stehen in gutem Einklang mit den bekannten Daten (Krismann M, Adams H u. Mitarb. 2000 [6]; Krismann M u. Mitarb. 2000 [8]).

Bisher sind aber weder spezifische Defekte noch bestimmte reproduzierbare Kombinationen von zytogenetisch fassbaren Defekten bei Mesotheliomen ermittelt worden.

\section{Zelluläre Reaktionen}

Bei der Diskussion zur Pathogenese maligner Mesotheliome kommt der Makrophagen-vermittelten Entzündungsreaktion eine wichtige Bedeutung zu. Die pulmonale und pleurale Deposition und Inkorporation von Asbestfasern führt nach in-vitroBefunden zu einer Aktivierung von Lymphozyten und Makrophagen. Eine klonale T-Helferzell-Expansion bewirkt durch vermehrte Zytokinsekretion eine Immunglobulinsekretion durch B-Lymphozyten. Kaskadenartig erfolgt eine Freisetzung von Tumor-Nekrosefaktor $\alpha$, Platelet derived growth factor (PDGF) sowie Fibronektin durch Makrophagen. Reaktive Sauerstoffmetaboliten werden vermehrt gebildet. Andererseits wird durch Interferon $\gamma$ die Kollagenfasersynthese in den Fibroblasten sowie die PDGF-induzierte Fibroblastenproliferation reduziert, was einen gewissen protektiven Aspekt darstellt. Die erhöhte Immunglobulinsekretion bewirkt an den Makrophagen eine gesteigerte Freisetzung von Sauerstoffradikalen bei Kontakt mit den Fasern und perpetuiert die Entzündungskaskade. Durch Sauerstoffradikale werden DNA-Schäden (Mutationen, Strangbrüche) ermöglicht. Eine detaillierte Übersicht zu den asbestassoziierten immunologischen Phänomenen findet sich bei Kagan und Brody (Kagan M, Brody AR 1996 [5]) (Abb. 5).

Ein aktueller Schwerpunkt liegt in der Erforschung der TumorAngiogenese. Dabei konnte eine Expression des angiogenen Zytokins VEGF (vascular endothelial growth factor) auf Proteinund mRNA-Ebene dokumentiert werden. Darüber hinaus konnte auch die Expression des VEGF-Rezeptors flt-1 (fms-like tyrosine kinase) ebenfalls auf mRNA- und Proteinebene, vorwiegend bei epitheloiden, teils aber auch bei biphasischen und sarkomatösen Mesotheliomen belegt werden. Dieser Rezeptor ist bei der Endothelorganisation und Monozytenmigration beteiligt. Aus der möglichen Blockierung von VEGF-Rezeptoren im Hinblick auf antiangiogenetische Auswirkungen ergeben sich Therapieansätze.

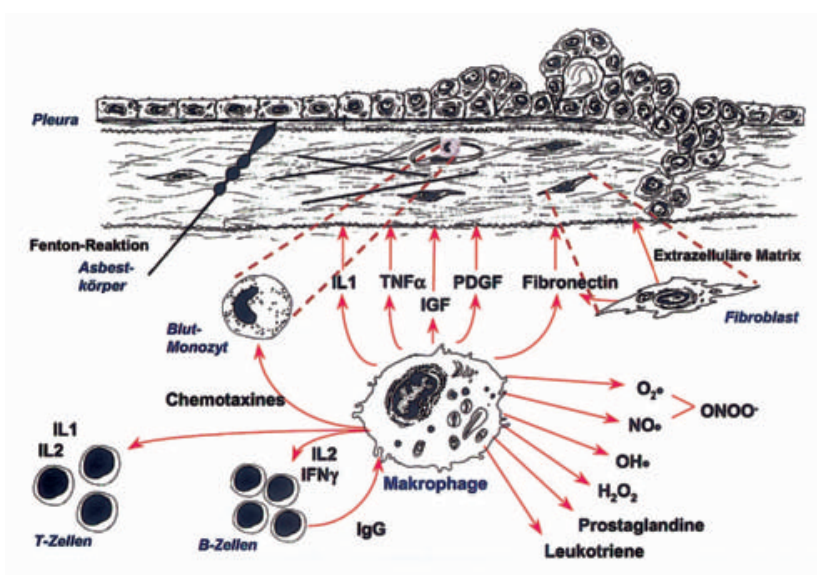

Abb. 5 Schema komplexer entzündlich-immunologischer Prozesse bei Asbest-assoziierten pleuro-pulmonalen Erkrankungen. Aktivierung von Alveolarmakrophagen und T-Zellen mit Freisetzung von Mediatoren.

\section{Pathologie}

\section{Makroskopische Befunde}

Vorgeschrittene Tumorausbreitungsphasen maligner Mesotheliome sind in der Regel makroskopisch durch ein charakteristisches Wachstumsmuster gekennzeichnet, das computertomographisch/radiologisch oder autoptisch wegweisend ist. Dennoch sind spezifische makroskopische Befunde nicht zu erheben (Abb. 6, Abb. 7).

Einerseits können Pleurakarzinosen anderer Primärtumoren pseudomesotheliomatöse Wachstumsmuster induzieren, andererseits können maligne Mesotheliome selten auch umschrieben ausgebildet sein. Bei den pleuralen Mesotheliomen handelt es sich in fortgeschrittenen Ausbreitungsphasen um ein mantelförmig, meist recht gleichmäßig die Lunge umschließendes, bevorzugt basal akzentuiertes weißes, wechselnd derbes, teils pseudozystisches Fremdgewebe. Dieses folgt charakteristischerweise

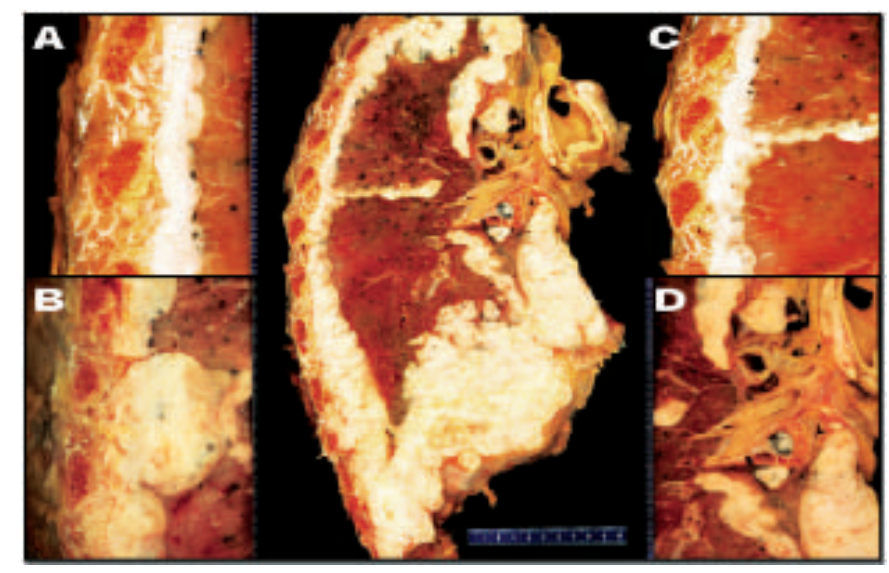

Abb. 6 Makroskopische Befunde eines weit vorgeschrittenen, vorwiegend sarkomatoid wachsenden Pleuramesothelioms in frontaler Schnittführung durch die rechtsseitige Thoraxregion.

a) und b) Mantelförmige, teils auch knotige Tumorentwicklung in der Grenzzone von Thoraxwand und Lunge.

c) Tumorpropagation entlang eines interlobären Septums.

d) Multiple Lymphknotenmetastasen im parahilären Bereich (62-jähriger Patient mit beruflicher Asbest-Exposition. Lungenstaubanalyse mit maximal 60 Asbestkörpern). 


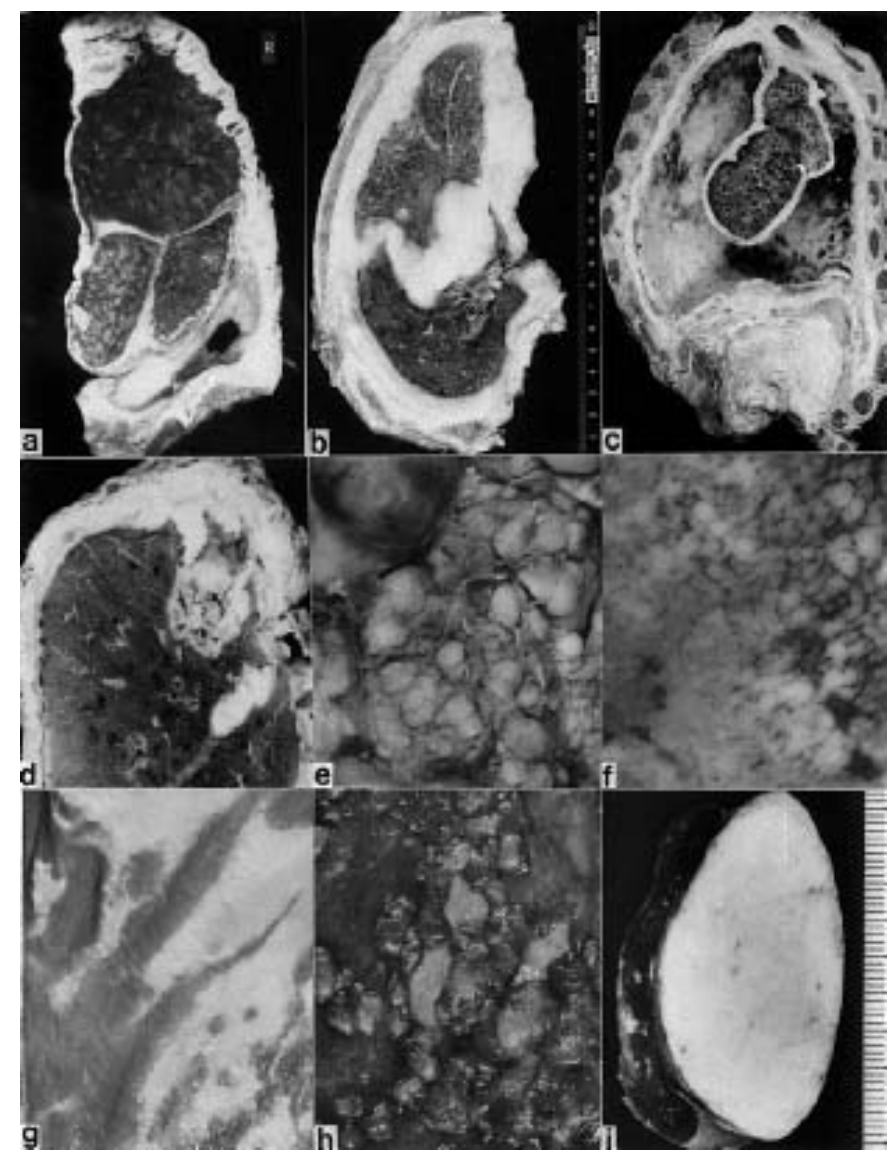

Abb. 7 Makroskopische Befunde pleuraler Neoplasien und hyaliner Pleuraplaques.

a) Frontalschnitt der rechten Lunge im Endstadium eines diffusen malignen Pleuramesothelioms. Basale ausgedehnte Ergusshöhle. Tumorpropagation entlang der interlobären Septen.

b) Horizontalschnitt der rechten Lunge $12 \mathrm{~cm}$ unterhalb der Spitze. Massive Tumorpropagation auch im mediastinalen und interlobären Bereich.

c) Hemithorax vom Bild des Endstadiums eines malignen Mesothelioms. Massive Kompression und Atelektase der hiluswärts komprimierten Restlunge durch einen massiven tumorbedingten Pleuraerguss.

d) Pseudomesotheliom als Folge der Pleurakarzinose eines peripheren Adenokarzinoms im Lungenoberlappen.

e) Nodulär wachsendes Pleuramesotheliom der parietalen Pleura.

f) Granuläre Pleurakarzinose bei einem kleinzelligen bösartigen Lungentumor.

g) Typische hyaline Pleuraplaques nach Asbeststaub-Exposition.

h) Noduläre pleurale Karzinose eines kleinzelligen bösartigen Lungentumors in Kombination mit kleinen typischen hyalinen Pleuraplaques. i) Lokalisierter gutartiger fibröser Pleuratumor, ausgehend von der Pleura pulmonalis mit schmalem anhängenden Saum und atelektatischem Lungengewebe (Operationspräparat).

den Interlobärspalten und führt zu einer Obliteration der komplementären Pleurablätter. Erst spät, besonders bei dem sarkomatoiden Subtyp, kommt es auch zur Infiltration in das angrenzende Lungenparenchym. In späten Stadien kommen auch multiple intrapulmonale Tumorknoten vor. Klinische Leitsymptome sind therapierefraktäre bzw. rezidivierende Pleurergüsse, Dyspnoe sowie thorakale Schmerzen. Die derzeit aktuelle TNM-Klassifikation der Pleuramesotheliome und die klinische Stadieneinteilung sind in Abb. 8 und Tab. 1 dargestellt (Hermanek P. u. Mitarb. 1997 [3], Rusch VW 1996 [11]).

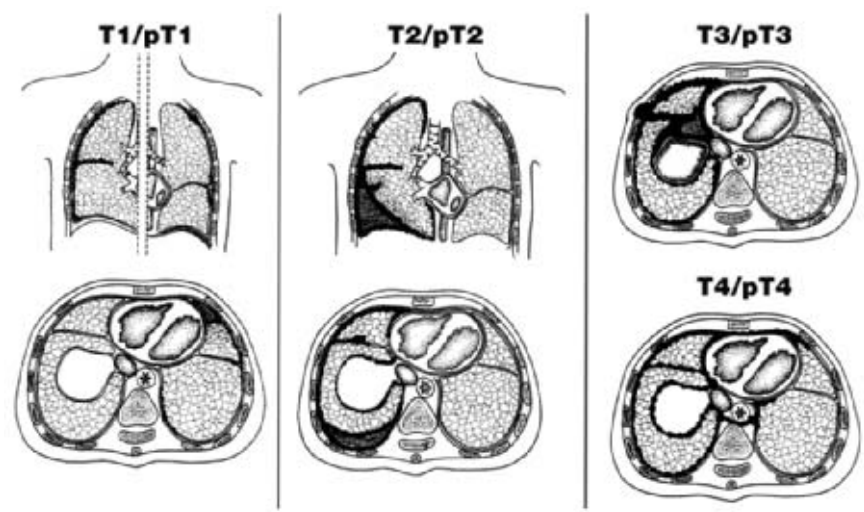

Abb. 8 Schematische Darstellung unterschiedlicher Tumorstadium beim Pleuramesotheliom nach der TNM-Klassifikation von 1999.

In frühen Wachstumsphasen finden sich bevorzugt in der Pleura parietalis, zum Diagnosezeitpunkt fast immer auch in der Pleura visceralis kleine weißliche fast multifokal ausgebildete Knötchen.

Ein Befall des Perikards oder Epikards kann selten primär, viel häufiger sekundär auftreten. Hier führt die ergussbedingte Kompression des Herzens mit zunehmender Ventrikelfunktionsstörung zur diagnostischen Punktion oder Probenentnahme.

\section{Histologische Befunde}

Histologische und zytologische phänotypische Bilder häufiger pleuraler und seltener peritonealer und perikardialer Mesotheliome sind äußerst variabel (Abb. 9).

Die differenzialdiagnostische Abgrenzung von noch reaktiven Mesothelproliferaten [RMH] über die atypische mesotheliale Hyperplasie [AMH], das Mesothelioma in situ [MIS] und Frühmesotheliome bis zu manifesten infiltrierenden, Pleura-überschreitenden Tumoren erfordert gute Kenntnisse und Erfahrungen des Pathologen und ausreichendes Untersuchungsgut (Abb. 10).

Nach führenden histologischen Wachstumstypen werden - entsprechend der 1999 revidierten WHO-Klassifikation der Lungenund Pleuratumoren - überwiegend epitheloide, überwiegend sarkomatoide und biphasische Mesotheliome unterschieden. Die Mehrzahl der malignen Mesotheliome weist eine biphasische Differenzierung auf, sofern größere Proben des Tumors untersucht werden. Die abschließende Bestimmung des histologisch phänotypisch führenden bzw. in der Regel in Kombination vorliegenden Wachstumstyps ist daher nur am Operationsgut oder im Rahmen einer Obduktion zu stellen. Anders als bei malignen Lungentumoren und zahlreichen anderen Organtumoren existiert für die Mesotheliome bislang kein verbindliches histopathologisches Gradingschema. Vom Pathologen sollte zunächst das phänotypisch führende histologische Wachstumsmuster und der Anteil des niedrigsten Differenzierungsgrads unter Berücksichtigung zellulärer Atypien, Mitosen sowie Nekrosen angegeben werden.

\section{Varianten}

Neben den 3 histologisch bestimmbaren Hauptgruppen sind noch mehrere seltene Varianten bekannt, die sich jeweils durch eine besondere Histomorphologie auszeichnen. Dazu gehört die 
Tab. 1 Internationales TNM- und klinisches Staging-System für Pleuramesotheliome unter Berücksichtigung der Subklassifikation im Stadium T1a (begrenzt auf Pleura parietalis) und 1b (Pleura parietalis und visceralis) (nach Rusch 1996)

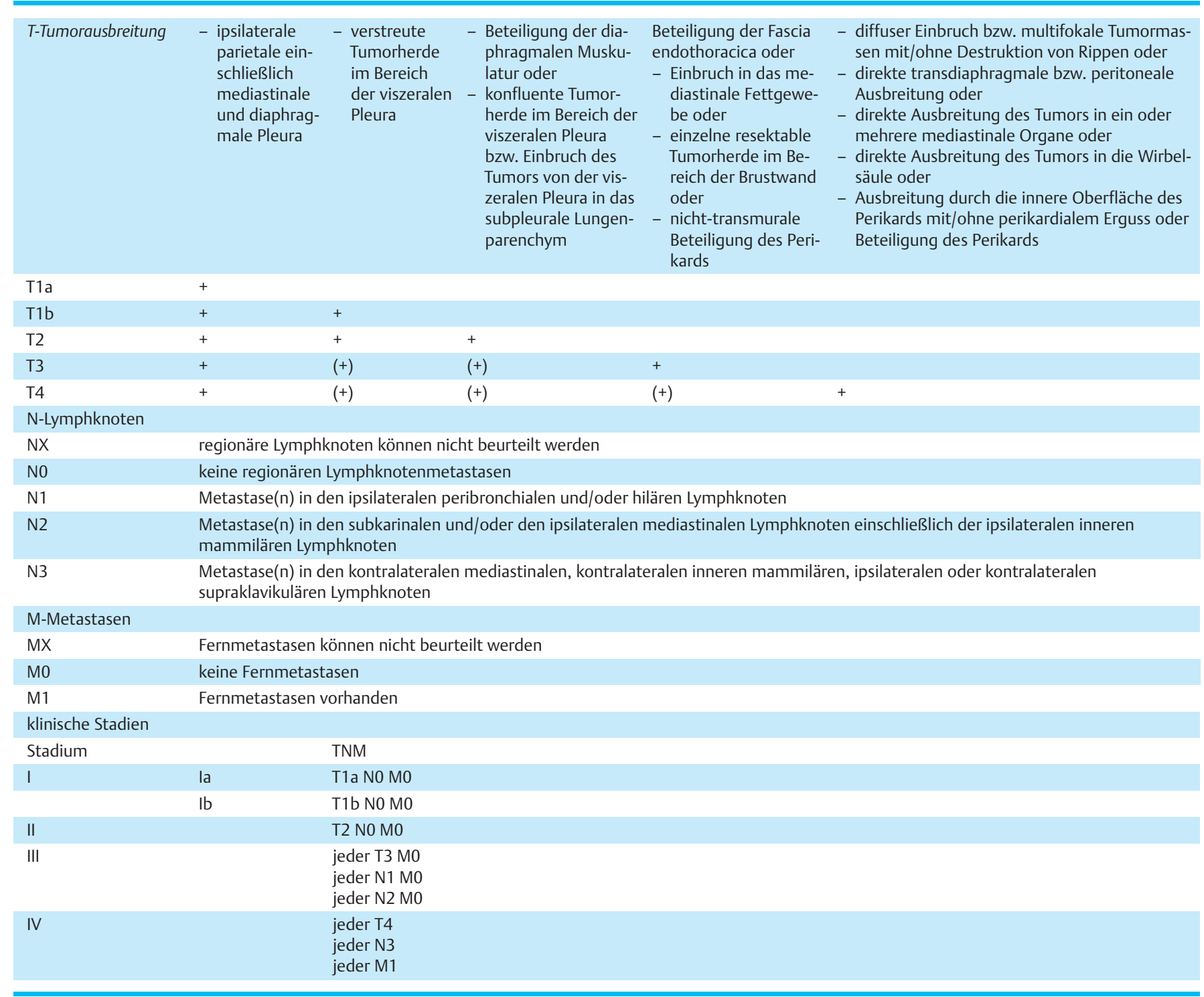

Rusch, V. W. A proposed new International TNM Staging System for malignant pleural mesothelioma from the International Mesothelioma Interest Group. Lung Cancer 14:1-12, 1996

in der WHO-Klassifikation aufgeführte desmoplastische Form, die im Einzelfall bei kleinen Biopsien erhebliche differenzialdiagnostische Schwierigkeiten bei der Abgrenzung gegenüber Pleuraschwarten bereiten kann, sowie das in der WHO-Klassifikation nicht gesondert angeführte deziduoide Mesotheliom und kleinzellige Varianten.

\section{Zusatzuntersuchungen}

Im Laufe der letzten Jahre ist die pathologisch-anatomische Differentialdiagnostik primärer und sekundärer Tumoren der serösen Häute durch den Ausbau und Einsatz der immunhistochemischen Verfahren erheblich bereichert worden. Die histologische Diagnose eines malignen Mesothelioms nach konventionellen lichtmikroskopischen und üblichen histochemischen Verfahren sollte grundsätzlich durch gezielt ausgewählte immunhistochemische Untersuchungen bestätigt werden (Tab. 2).
Es konnten zwar charakteristische wiederkehrende, bisher aber keine spezifischen Befunde für Mesotheliome aufgezeigt werden.

Es gibt 3 klassische differenzialdiagnostische Probleme in der Mesotheliomdiagnostik. Sekundäre Pleurakarzinosen von Adenokarzinomen können ähnliche Bilder hervorrufen wie vorwiegend epitheloide Mesotheliome. Zur Differenzialdiagnose wird hier üblicherweise in Ergänzung der histochemischen PAS-Färbung ohne und mit Diastasevorbehandlung zum Schleimnachweis ein immunhistochemisches Markerpanel eingesetzt. Eine eindeutige Mesotheliomdiagnose kann bei zellulärer Koexpression von Keratinen und Vimentin sowie Nachweis von Calretinin, bei negativer Reaktion mit dem Antikörper gegen HEA und Fehlen diastaseresistenter Vakuolen erfolgen (Müller K-M 1997 [10]; Travis WD u. Mitarb. 1999 [13]). Es gibt aber Grenzfälle, bei denen einzelne Details nicht eindeutig zur Diagnose passen. Bei 


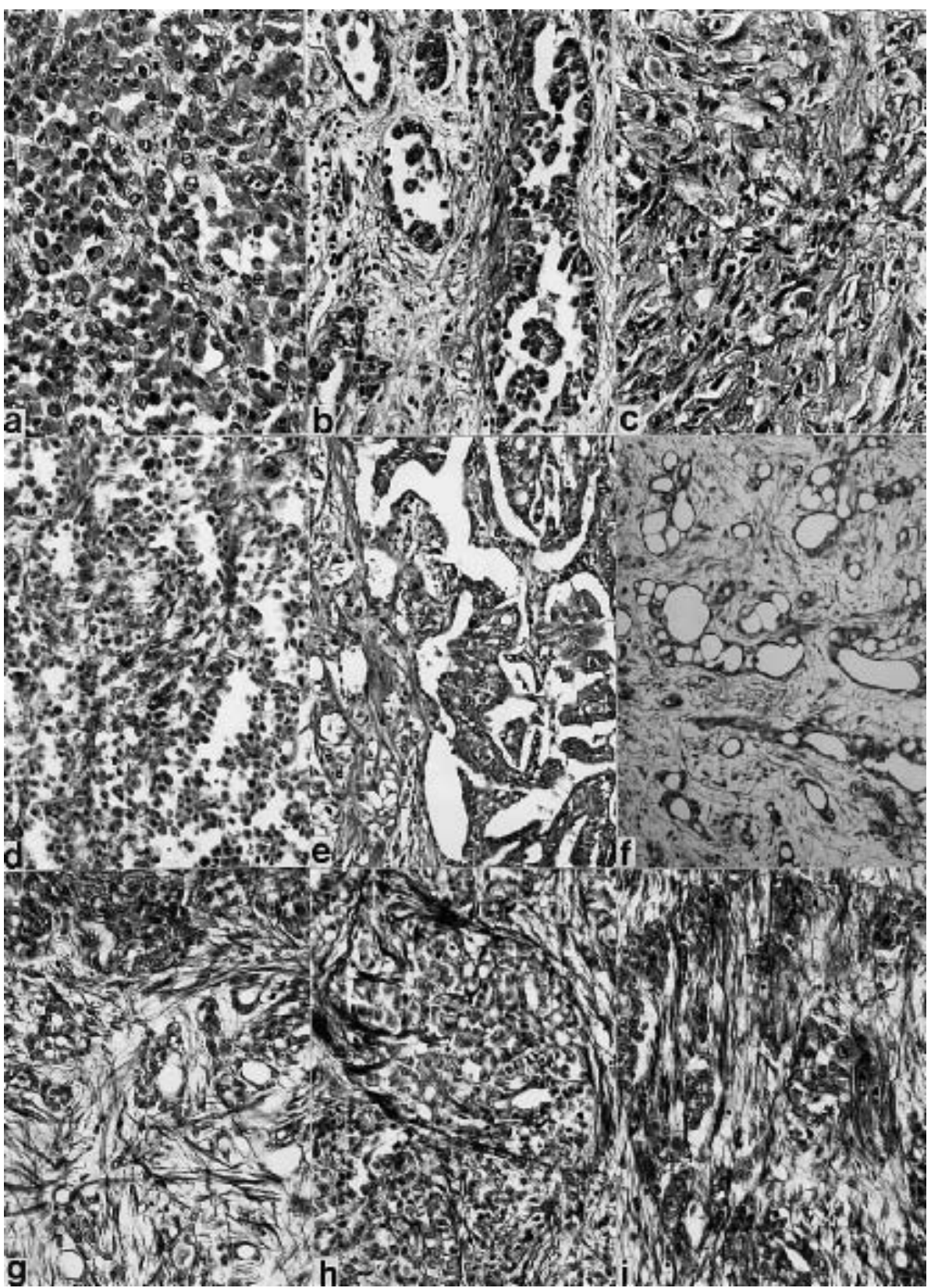

Abb. 9 Mikrofotogramme mit Beispielen verschiedenartiger Wachstumsmuster von Pleuramesotheliomen.

a) - b) Hoch differenziertes epitheloides Mesotheliom der Pleura parietalis in einer frühen Entwicklungsphase mit beginnender Infiltration von subpleuralem Fettgewebe (so genanntes Frühmesotheliom $60 \times / 150 \times)$

c) -f) Ausschnitte von vier Arealen des selben Mesothelioms. Adenoide/epitheloide (c, d)>, biphasische (e, f) Differenzierungen (jeweils 150×)

g) -i) Sarkomatoid-pleomorphes, niedrig differenziertes Mesotheliom mit hochgradigen Zell- und Kernatypien (150×/250×).
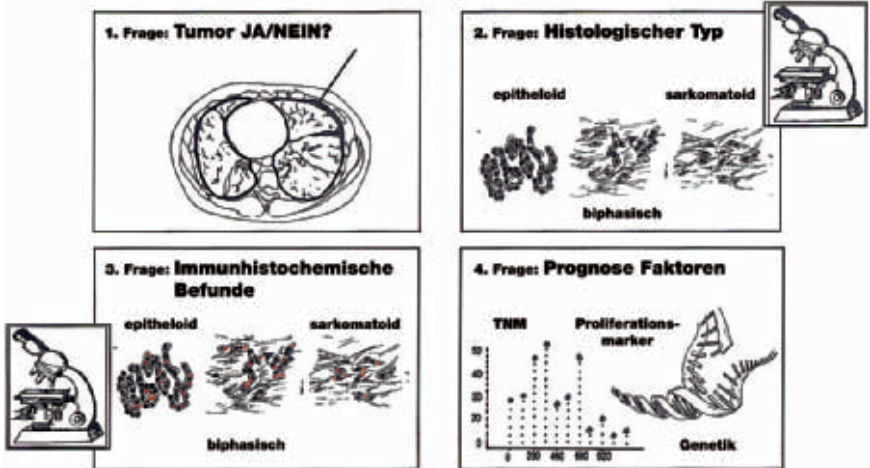

Abb. 10 Aufgaben des Pathologen bei der Differenzierung reaktiver und neoplastischer, primärer und sekundärer Pleuraerkrankungen. derartigen Problemfällen muss das Krankheitsbild im Rahmen eines klinisch-pathologischen Konsils - auch unter Berücksichtigung versicherungsmedizinischer Fragen - erörtert werden.

Die Abgrenzung sarkomatoider Mesotheliome von Pleurasarkomatosen primärer Weichteilsarkome gelingt in der Regel mit Hilfe der in der Sarkomdiagnostik eingesetzten Antikörper. Vorwiegend sarkomatoide Mesotheliome koexprimieren meist Keratine und Vimentin, seltener sind auch Calretinin und Aktin nachweisbar.

Ausgesprochen problematisch kann im Einzelfall die differenzialdiagnostische Abgrenzung reaktiver Serosaläsionen von frühen neoplastischen Befunden sein (Abb.11).

Bei dieser Problematik kann auch die immunhistochemische Untersuchung gelegentlich nicht abschließend weiterführen. Man ist auf die Beurteilung zytologischer Atypien und eines invasiven 
Tab. 2 Zusammenstellung charakteristischer, aber nicht spezifischer immunhistochemisch fassbarer Befunde zur Differenzialdiagnose primärer und sekundärer bösartiger Tumoren im Bereich der serösen Häute

\begin{tabular}{|c|c|c|c|c|}
\hline \multirow[t]{2}{*}{ Antikörper } & \multicolumn{2}{|c|}{ Pleuramesotheliome } & \multirow{2}{*}{$\begin{array}{l}\text { Pleurakarzi- } \\
\text { nosen }\end{array}$} & \multirow{2}{*}{$\begin{array}{l}\text { Pleurasar- } \\
\text { komatosen }\end{array}$} \\
\hline & Epitheloid & Sarkomatoid & & \\
\hline Calretinin & +++ & + & $(+)$ & - \\
\hline $\begin{array}{l}\text { MNF116 } \\
\text { Zytokeratine }\end{array}$ & +++ & ++ & ++ & + \\
\hline $\begin{array}{l}\text { AE1/AE3 } \\
\text { Zytokeratine }\end{array}$ & ++ & ++ & ++ & + \\
\hline CK5/6> & ++ & - & $(+)$ & - \\
\hline BMA120 & ++ & $(+)$ & - & - \\
\hline V9 Vimentin & ++ & +++ & + & +++ \\
\hline EMA & $\begin{array}{l}++ \\
\text { membranös }\end{array}$ & $(+)$ & $\begin{array}{l}+ \\
\text { cytoplasma- } \\
\text { tisch }\end{array}$ & - \\
\hline BerEP4 HEA & $(+)$ & - & +++ & + \\
\hline CD 15 & - & - & + & - \\
\hline CEA & - & - & ++ & - \\
\hline TTF1 & - & - & ++ (Lunge) & - \\
\hline Aktin & $(+)$ & $(+)$ & - & + \\
\hline Desmin & $(+)$ & $(+)$ & - & + \\
\hline Myogenin & - & - & - & + \\
\hline CD 99 & - & - & - & + \\
\hline S-100 & - & - & - & + \\
\hline SMA & $(+)$ & +++ & - & - bis +++ \\
\hline
\end{tabular}

[+++ = regelmäßig positiv; ++ = häufig positiv; + = gelegentlich positiv;

$(+)=$ gelegentlich fokal positiv, $-=$ in der Regel negativ]

Wachstums angewiesen. In Biopsien gibt es gelegentlich Befunde oberflächlicher nicht invasiver Neoplasien, die im Sinne eines sog. Mesothelioma in situ eingeordnet werden müssen und zusammen mit den minimal-invasiv wachsenden Mesotheliomen unter dem Begriff des Frühmesothelioms zusammengefasst werden. Einerseits können reaktive Pleuraläsionen, z.B. im Rahmen einer Pleuritis bei rheumatischer Grunderkrankung, mäßiggradige nukleäre Atypien aufweisen, andererseits gelingt bei bereits neoplastischen toporegional unterschiedlich weit fortgeschrittenen Läsionen in Frühstadien oft der Nachweis eines infiltrativen Wachstums noch nicht (Abb.11).

Weiterführende Verfahren unter Einsatz molekularpathologischer Methoden befinden sich derzeit noch in der Erprobung.

Der zuweilen problematischen Differenzialdiagnose Rechnung tragend, hat das Europäische Mesotheliompanel 1993 eine 5stufige Klassifikation nach histologischen Kriterien für die diagnostische Sicherheit etabliert, die immer noch Gültigkeit besitzt. Dabei bedeutet die Kategorie
A sicheres,
B wahrscheinliches,
C mögliches Mesotheliom
D wahrscheinlich und
E sicher kein Mesotheliom.

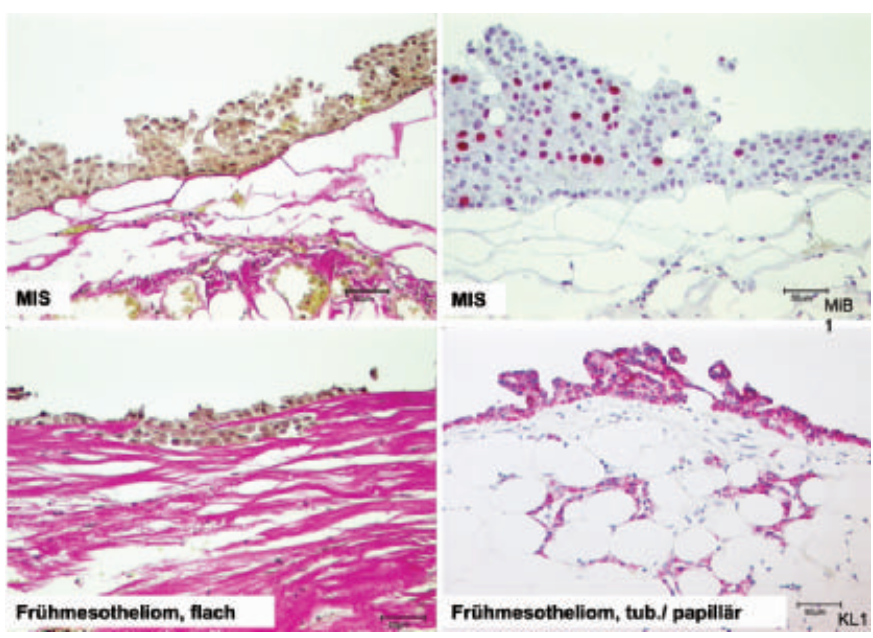

Abb. 11 Histologische Befunde zur Differenzialdiagnose von Mesothelioma in situ und Frühmesotheliom.

Für versicherungsmedizinische Fragestellungen einer Berufskrankheit der Ziffer 4105 der Berufskrankheitenverordnung sind die Kategorien A und B als diagnostische Sicherheit ausreichend.

\section{Stellenwert molekularpathologischer Zusatzuntersuchungen}

Derartige Untersuchungen ergänzen derzeit die Diagnostik, aufgrund bislang fehlender spezifischer Defekte erlangen sie aber nicht den Status diagnosesichernder Untersuchungen im Spektrum der Differenzialdiagnose primärer und sekundärer maligner Erkrankungen der serösen Häute. Da genetische Tumoranalysen zeit- und kostenaufwendig sind, ist Ihr Einsatz im Rahmen der täglichen Begutachtungspraxis derzeit nicht zu gewährleisten.

\section{Versicherungsmedizinische Aspekte von Pleura- erkrankungen}

Das maligne diffuse Pleuramesotheliom, Pleuraplaques der Pleura parietalis und die so genannte diffuse Pleurafibrose der Pleura pulmonalis gewinnen unter versicherungsmedizinischen Aspekten besondere Bedeutung.

Bei der Bewertung eines wahrscheinlichen Kausalzusammenhanges zwischen einer bekannten beruflichen Asbestexposition und einem bösartigen Lungentumor werden die Befunde hyaliner Pleuraplaques und der klinisch schwer fassbaren diffusen Fibrose der Pleura pulmonalis, auch in Kombination mit so genannten Rundherdatelektasen als morphologische Indizien einer wahrscheinlich erhöhten Asbestbelastung bei der Zusammenhangsbegutachtung berücksichtigt. Die Erkrankungen sind in der Anlage 1 zur Berufskrankheitenverordnung vom 18.12.1994 unter der Nr. 4: Erkrankungen der Atemwege und der Lungen, des Rippenfells und des Bauchfells, hier bei der Unternummer 41: „Erkrankungen durch anorganische Stäube“ zusammengefasst. Die zuständigen Berufskrankheitsziffern und ihre Bezeichnungen lauten:

- BK-Ziffer 4104: Lungenkrebs in Verbindung mit Asbeststaublungenerkrankungen (Asbestose) oder mit durch Asbeststaub verursachten Erkrankungen der Pleura (hyaline Plaques der Pleura parietalis und diffuse Pleuraverdickung der Pleura pulmonalis) oder bei Nachweis der Einwirkung einer kumulativen Asbestfaserstaubdosis am Arbeitsplatz von mindestens 25 Faserjahren $\left(25 \times 10^{6}\left(\right.\right.$ Fasern $/ \mathrm{m}^{3} \times$ Jahre $\left.)\right)$. 
- BK-Ziffer 4105: Durch Asbest verursachtes Mesotheliom des Rippenfells, des Bauchfells und des Herzbeutels.

In der Zeit von 1978-2000 sind in Deutschland (BRD) 6626 Pleuramesotheliome als Asbest-bedingte Berufskrankheiten von den verschiedenen Berufsgenossenschaften anerkannt worden (Butz M 2002 [1]). Dies ist ein Anteil von 32,9\% der Gesamtzahl beruflich bedingter Krebserkrankungen. 53,9\% (10877) entfallen auf überwiegend Asbest-assoziierte Lungentumoren. In den vergangenen 15 Jahren ist ein fast kontinuierlicher Anstieg der Asbest-bedingten Pleuramesotheliome zu verzeichnen (Butz M 2002 [1]).

- Eine relativ große Diskrepanz zwischen den pro Jahr angezeigten begründeten Verdachtsfällen und den entschädigten Fällen gründet sich zunächst auf die oft schwierige Differenzialdiagnose zwischen Mesotheliomen und sekundären Pleuratumoren bzw. reaktiven Pleuraveränderungen.

- Außerdem sind für die haftungsbegründende Kausalität einer Berufskrankheit nach Ziffer 4105 die Objektivierung des bösartigen Pleuratumors und die Ermittlung einer stattgehabten beruflichen Asbeststaubexposition Voraussetzung.

- Die haftungsausfüllende Kausalität kann durch ergänzende staubanalytische Untersuchungen mit Quantifizierung der Asbestfasern bzw. Asbestkörperchen erfolgen. Für diese Untersuchungen ist aber repräsentatives Lungengewebe (Lungenwürfel von mindestens $1 \mathrm{~cm}^{3}$ Größe) erforderlich. In autonom wachsenden bösartigen Pleura- und Lungentumoren liegt eine Anreicherung von Asbestfasern in der Regel nicht vor.

Im Bochumer Mesotheliomregister ließen sich bei den gesicherten malignen Pleuramesotheliomen anhand der Ergebnisse der Lungenstaubanalysen in über $90 \%$ Anhaltspunkte für eine vermehrte, meist beruflich bedingte Asbestexposition gewinnen. Bemerkenswert in diesem Zusammenhang ist das Familienschicksal eines Asbestarbeiters, dessen Arbeitskleidung zu Hause von der Ehefrau gereinigt wurde. Vater, Mutter und Sohn erkrankten an einem diffusen malignen Pleuramesotheliom, wobei die Erkrankung bei dem Sohn bereits im Alter von 41 Jahren aufgetreten ist.

\section{Sekundäre Pleuratumoren}

Sekundäre metastatische Neubildungen im Bereich der Pleura sind wesentlich häufiger als primäre Pleuratumoren. Bei einer gegenwärtigen jährlichen Anzahl von ca. 800 Todesfällen an malignen Mesotheliomen lassen sich ca. 8500 sekundäre bösartige Pleuratumoren berechnen. Andere Schätzungen gehen von 30000 Pleurakarzinosen bzw. -sarkomatosen pro Jahr aus.

Die frühzeitige Mitbeteiligung der Pleura bei primären pulmonalen oder extrapulmonalen Tumoren erklärt sich durch die reichhaltige Entwicklung pleuraler Lymph- und Blutgefäße. In vorgeschrittenen Phasen von Lungentumoren ist eine Mitbeteiligung der Pleura in $40 \%$ vorhanden. Dies gilt besonders für Adenokarzinome und kleinzellige Karzinome.

Bei Plattenepithelkarzinomen ist eine Pleurakarzinose eher selten. Pleuraergüsse sind bei diesem Tumortyp häufiger Folgen unspezifischer Pleurareizungen besonders durch chronische Retentionspneumonien.
Die häufigsten Primärtumoren mit sekundärer Pleurabeteiligung sind bei Männern und zunehmend häufiger bei Frauen primäre Lungentumoren (besonders Adenokarzinome und kleinzellige Karzinome), gefolgt von Mammatumoren und bösartigen Primärtumoren von Ovar, Uterus, Magen-Darm-Trakt, Pankreas, Nieren und Leber. Auch vorwiegend peripher im Lungengewebe angeordnete hämatogene Metastasen von Nierenkarzinomen, Dickdarmkarzinomen, Osteosarkomen, Weichteilsarkomen und Melanomen führen zu einer frühzeitigen Mitbeteiligung der Pleura pulmonalis. In fortgeschrittenen Metastasierungsphasen können breite pleurale Tumorschwarten gelegentlich makroskopisch nicht sicher von einem primären malignen diffusen Mesotheliom abgegrenzt werden.

\section{Histologie und Differenzialdiagnose sekundärer Pleuratumoren}

Ein wichtiger morphologischer Hinweis auf eine sekundäre pleurale Blastomatose ist das Vorkommen von Tumorzellen im Bereich intrapleuraler Lymph- und Blutgefäße. Die begleitende unspezifisch-entzündliche Reaktion erklärt pleurale Begleitergüsse ohne den zytologischen Nachweis von Tumorzellen. Im Erguss sind aber fast regelmäßig aktivierte Mesothelzellen und Subserosazellen vorhanden, die bei erheblicher Zell- und Kernpolymorphie die zuverlässige Abgrenzung zu einem Pleuramesotheliom erschweren. Bei der Abgrenzung möglicher Metastasierungen primärer Prostata- oder Schilddrüsentumoren sind gelegentlich immunhistochemische Marker hilfreich.

\section{Differenzialdiagnostische Probleme}

Bei bisher nur im Ansatz vorhandenen therapeutischen Maßnahmen unter kurabler Zielsetzung muss die Diagnostik auf die Erfassung von Mesotheliomen in frühen Erkrankungsphasen ausgerichtet werden. In mehreren Fällen konnten im Mesotheliomregister in den letzten Jahren pleurale Läsionen im Stadium eines so genannten Frühmesothelioms mit noch auf oberflächliche Pleurazonen beschränktem Wachstum erfasst werden.

\section{Prognostische Parameter}

Grundsätzlich gehören die Mehrzahl der MDM in die Kategorie der Tumoren mit geringer Langzeitüberlebenschance. Derzeit wird aktiv an multimodalen Therapiekonzepten gearbeitet, eine standardisierte Therapie mit potenziell kurativem Ansatz ist aber insgesamt noch nicht in Sicht. Nach wie vor werden die meisten Mesotheliome erst in vorgeschrittenen Stadien entdeckt. An der mittleren Überlebenszeit von etwa 9-12 Monaten nach Erstdiagnose hat sich auch in zahlreichen Studien mit operativen und chemotherapeutischen Verfahren prinzipiell nichts geändert. Erste Erfolge mit multimodalen Konzepten müssen an größeren Kollektiven bestätigt werden. Etwa 10\% der Mesotheliome weisen jedoch auch therapeutisch weitgehend unbeeinflusst einen mehrjährigen Verlauf auf. Epitheloides Wachstumsmuster, hoher so genannter Performance status (guter Allgemeinzustand bei Erstdiagnose), jüngeres Alter, frühes TNM-Stadium, starke myxoide/ödematöse Stromakomponente sowie niedrige Proliferationsaktivität (MiB1 < 10\%) sind Indikatoren einer in der Regel günstigeren Prognose. 
Besonders ungünstig hingegen sind sarkomatoides desmoplastisches Wachstum, geringe Differenzierung, hoher Proliferationsgrad (> 40\%), höheres Lebensalter und schlechter AZ bei Erstdiagnose.

\section{Literatur}

${ }^{1}$ Butz M. Beruflich verursachte Krebserkrankungen 1978-2000. 8. Auflage. Sankt Augustin: Hrsg. Hauptverband der gewerblichen Berufskrankheiten (HVgB), 2002

${ }^{2}$ Carbone M, Pass HI, Rizzo P et al. Simian virus 40-like DNA sequences in human pleural mesothelioma. Oncogene 1994; 9: 1781 - 1790

${ }^{3}$ Hermanek P, Hutter RVP, Sobin LH et al. UICC International Union Against Cancer TNM Atlas. 4th edition. Springer-Verlag: 1997

${ }^{4}$ Jones JSP, Brachet EA, Butler EB. The pleura and its pathology. In: Jones JSP (ed); Pathology of the mesothelium. Berlin Heidelberg, New York, Tokyo: Springer-Verlag, 1987: 39-133

${ }^{5}$ Kagan M, Brody AR. Immunopathology of asbestos-related lung disease In: Kradin RL, Robinson BWS (eds); Immunopathology of Lung diseases. Boston: Butterworth Heinemann, 1996: 421

${ }^{6}$ Krismann M, Adams H, Jaworska M et al. Patterns of chromosomal imbalances in benign solitary fibrous tumors of the pleura. Virchows Arch 2000; 437: 248-255
${ }^{7}$ Krismann M, Johnen G, Wiethege T et al. Pleurale Tumoren. In: Ganten D, Ruckpaul K (Hrsg.); Nicht-hereditäre Tumorerkrankungen. Berlin, Heidelberg: Springer-Verlag, 2002: 65-86

${ }^{8}$ Krismann M, Müller K-M. Malignes Mesotheliom der Pleura, des Perikards und des Peritoneums. Chirurg 2000; 71: 877-886

${ }^{9}$ Light RW. Pleural Diseases. $4^{\text {th }}$ ed. Philadelphia: Lippincott Williams \& Wilkins, 2001

${ }^{10}$ Müller K-M. Mesotheliome. Pneumologie 1997; 51: 335 - 344

${ }^{11}$ Rusch VW. A proposed new international TNM staging system for malignant pleural mesothelioma. From the international mesothelioma interest group. Lung Cancer 1996; 14: 1-12

12 Shivapurkar N, Wiethege Th, Wistuba II et al. Presence of simian virus 40 sequences in malignant pleural, peritoneal and non-invasive mesotheliomas. Int J Cancer 2000; 85: $743-745$

${ }^{13}$ Travis WD, Colby TV, Corrin B et al. Histological typing of lung and pleural tumors. 3rd edition. Berlin: Springer-Verlag, 1999

${ }^{14}$ Wagner JC, Sleggs CA, Marchand P. Diffuse pleural mesothelioma and asbestos exposure in the North Western Cape Province. Br J Ind Med 1960; 17: $260-271$

\section{Bereits publizierte Beiträge zu dieser Serie:}

${ }^{1}$ Bildgebende Diagnostik bei Pleuraerkrankungen. Pneumologie 2004; 58: $238-254$

2 Pleura: Pathologie nicht-neoplastischer Erkrankungen. Pneumologie 2004; 58: 516-524 